\title{
The levels of sMUC-1 in patients with multiple myeloma
}

\section{Dorota Lemancewicz ${ }^{1,2}$, Lukasz Bolkun², Halina Porowska ${ }^{3}$, Marzenna Galar², Janusz Semeniuk $^{4}$, Janusz Kloczko ${ }^{2}$, Janusz Dzieciol ${ }^{1}$}

\author{
${ }^{1}$ Department of Human Anatomy, Medical University of Bialystok, Poland \\ ${ }^{2}$ Department of Hematology, Medical University of Bialystok, Poland \\ ${ }^{3}$ Department of Medical Chemistry, Medical University of Bialystok, Poland \\ ${ }^{4}$ Department of Immunology and Pediatrics Gastroenterology and Allergology, \\ Medical University of Bialystok, Poland
}

\begin{abstract}
Mucins have been shown to be aberrantly overexpressed in various diseases including cystic fibrosis, asthma, and cancer. Recent studies have uncovered the roles of these mucins in the pathogenesis of cancer. The presence of MUC-1 has also been detected on the cell surface of multiple myeloma (MM) cells in peripheral blood and showed direct correlation with tumor mass. In this study, we evaluated the levels of soluble MUC-1 (sMUC-1) in 50 new MM patients and correlated this with the levels of sMUC-1 after treatment. High levels of sMUC-1 were found in 20/50 (40\%) MM patients, and in 2/50 (4\%) healthy individuals $(\mathrm{p}=0.001)$. According to the ISS, we found significant differences of mean sMUC-1 levels between the first stage of the disease $(0.63 \pm$ $\pm 0.26)$ and the third $(0.93 \pm 0.24 ; p=0.03)$, but not with the second stage $(0.80 \pm 0.22 ; p=0.08)$. Our study confirmed the correlation between elevated sMUC-1 and high elevated lactate dehydrogenase $(p=0.03)$ and the level of $\operatorname{IgG}$ in groups of patients with MM IgG at every stage of disease $(p=0.001)$. We showed for the first time that levels of sMUC- 1 after treatment, in a group of patients with initially elevated levels of MUC-1, were statistically lower than in a group of patients with initially lower levels of sMUC-1 (21\% vs. $42,6 \%$; $=0.05)$. At 37 months median of follow-up, we found a statistically significant difference between patients with normal versus elevated sMUC-1 in terms of progression-free survival (median 12 months vs. 8.1 months; $\mathrm{p}=0.03$ ). (Folia Histochemica et Cytobiologica 2011; Vol. 49, No. 4, pp. 654-658)
\end{abstract}

Key words: multiple myeloma, sMUC-1

\section{Introduction}

MUC-1 is a highly glycosylated transmembrane protein, with high molecular weight. It is produced by ductal epithelial cells and normally presents on the luminal surface of secretory glands [1]. A soluble form of MUC-1 (sMUC-1, CA 15.3 CA27.29 antigen) has been found in breast milk, peripheral blood, urine,

Correspondence address: D. Lemancewicz,

Department of Human Anatomy,

Medical University of Bialystok,

Mickiewicza Str. 2A, 15-039 Bialystok, Poland;

e-mail:dorlem@poczta.onet.pl and supernatants from cultures of MUC- $1^{+}$cancer cell lines and primary cancer cells [1-4]. Increased levels of sMUC-1 have been detected in many adenocarcinomas, such as breast, ovary, pancreas and lung, and seem to correlate with tumor burden [5-7]. The level of sMUC-1 has been declared by the FDA (US Food and Drug Administration) to be a useful marker in monitoring disease activity for breast cancer patients. The function of MUC- 1 remains to be defined. From an immunologic standpoint, both an immunostimulatory [8-10] and an immunoinhibitory $[11,12]$ role have been proposed. MUC-1 disturbs cell-cell interaction, inhibits aggregation in vitro, and decreases the activity of natural killer cells and lym- 
phokine activated killer cells in cancer tissues [13-15]. Mucins have been shown to contain complex associations with various cellular pathways, impacting cell growth, proliferation, and apoptosis. Historically, transformation events in cancer have been defined as initiation events (contributing to the early stages of neoplastic transition) or progression events (referring to the subsequent transformative processes) [16]. Using human tumor xenograft models, MUC-1 has been shown to cause transformation of fibroblast cells. Also, the alteration of the tumorigenicity and metastasis of various cancer cell lines by overexpression and downregulation of mucins demonstrates their role in the pathogenesis of different malignancies [17-19]. Recent studies have suggested that MUC-1 modulates various pathways contributing to cell growth. MUC-1 directly binds to the p53 regulatory domain and selectively promotes transcription of growth arrest genes. It decreases transcription of apoptotic genes as a survival response to stress, and thereby decreases cell death [20].

Furthermore, recent studies have suggested that sMUC-1 may be an important immunosuppressive agent in patients with MUC-1-bearing malignancies, as evidenced by the poor response to active specific immunotherapy in patients with metastatic adenocarcinomas who have highly elevated sMUC-1 levels [21]. Therefore, determining sMUC-1 levels in multiple myeloma (MM) patients may be important from an immunobiologic standpoint.

The aim of this study was to estimate the level of sMUC-1, to explore whether there are differences in sMUC-1 level between patients with different stages of multiple myeloma and healthy individuals, and to assess the variation of sMUC-1 during chemotherapy treatment.

\section{Material and methods}

Fifty patients with newly diagnosed MM were studied. The median age of patients when the samples were obtained was 59 and the range was 40-75. Twenty four of them were female and 26 were male. The pre-treatment evaluation included a complete blood count, albumin in serum, the concentration of paraprotein in serum, immunoglobulin class subtype (Ig) and the levels of calcium, beta-2-microglobulin $\left(\beta_{2} \mathrm{~m}\right)$, lactate dehydrogenase $(\mathrm{LDH})$ and C-reactive protein (CRP). Bone marrow aspirates and trephine biopsies (TB) to detect plasma cells were obtained (Table 1). Patients at the time of diagnosis were allocated into one of three groups based on the International Staging System (ISS): five patients in the first stage, 20 in the second stage, and 25 in the third stage.

Patients' initial treatment for multiple myeloma depended on their age and comorbidities. For patients under the
Table 1. Characteristics of MM patients

\begin{tabular}{|l|c|}
\hline & Mean \pm SD \\
\hline Age & $70.82 \pm 9.90$ \\
\hline $\mathrm{Hb}[\mathrm{g} / \mathrm{dl}]$ & $10.56 \pm 2.16$ \\
\hline Serum Ca [mmol/L] & $2.46 \pm 0.29$ \\
\hline Serum M protein $[\mathrm{g} / \mathrm{dl}]$ & $2.37 \pm 2.17$ \\
\hline Plasma cells in aspirate $(\%)$ & $23.11 \pm 19.90$ \\
\hline Plasma cells in TB $(\%)$ & $45.18 \pm 28.52$ \\
\hline IgG $[\mathrm{mg} / \mathrm{dl}]$ & $3331.61 \pm 2371.16$ \\
\hline IgA [mg/dl] & $3260.64 \pm 2554.67$ \\
\hline IgM [mg/dl] & $43.67 \pm 44.82$ \\
\hline Serum albumin $[\mathrm{g} / \mathrm{l}]$ & $3.94 \pm 0.64$ \\
\hline$\beta 2 \mathrm{~m}[\mathrm{mg} / \mathrm{L}]$ & $5469.47 \pm 3804.62$ \\
\hline $\mathrm{CRP}[\mathrm{mg} / \mathrm{L}]$ & $6.57 \pm 4.53$ \\
\hline $\mathrm{LDH}[\mathrm{IU} / \mathrm{l}]$ & $201.71 \pm 46.90$ \\
\hline
\end{tabular}

$\mathrm{Hb}$ - hemoglobin; $\mathrm{Ca}$ - calcium; $\mathrm{M}$ - monoclonal; $\mathrm{TB}$ - trephine biopsy; Ig - immunoglobulin; $\beta 2 \mathrm{~m}$ - beta-2-microglobulin; $\mathrm{CRP}$ - C-reactive protein; $\mathrm{LDH}$ - lactate dehydrogenase

age of 65 , the induction regimens were thalidomide-dexamethasone and cyclophosphamide (CTD) prior to autologous stem-cell transplantation. For patients over 65 and patients with significant concurrent illness, the standard of care was chemotherapy with melphalan, prednisone and thalidomide (MPT). Of the 50 patients, 28 were treated with CTD combination therapy and 22 with MPT therapy. Response was assessed after six cycles of chemotherapy according to the European, International, and Autologous Bone Marrow Transplant Registries (EBMT/IBMTR/ (ABMTR) criteria [22].

Informed written consent was obtained from all participants and the Ethics Committee of the Medical University of Bialystok approved the study.

The control group consisted of 50 healthy individuals, age- and sex-matched.

Assay of MUC-1. Assay of MUC-1 was done according to the previously published protocol [23].

Samples of human serum were diluted in PBS to protein concentration $2 \mu \mathrm{g} / \mathrm{mL}$ and aliquots $(50 \mu \mathrm{L})$ were coated onto microtiter plates (NUNC F96; Maxisorp, Roskilde, Denmark) at room temperature overnight. The protein concentration was determined using the bicinchoninic acid method according to the manufacturer's instructions (BCA Protein Assay Kit, Pierce, Rockford, IL, USA). The wells were washed three times $(100 \mu \mathrm{L})$ with PBS, pH 7.4, $0.05 \%$ Tween (PBS-T; washing buffer) between all ensuing steps. Unbound sites were blocked with $100 \mu \mathrm{L}$ of $1 \%$ blocking reagent for ELISA (Roche Diagnostics, Mannheim, Germany) for $1 \mathrm{~h}$. The wells were than incubated for 2 hours 
with $100 \mu \mathrm{L}$ of anti-MUC-1 monoclonal antibody (anti-EMA, clone GP1,4; Sigma, St Louis, MO, USA) diluted in PBS-T/1\% BSA (Sigma) 1:500 (v/v). Next, plates were incubated ( $1 \mathrm{~h})$ with $100 \mu \mathrm{L}$ of secondary antibody, horseradish peroxidase conjugatated anti-mouse IgG (Sigma) diluted in PBS-T/1\% BSA 1:1,500 (v/v). Next, plates were washed four times in PBS, and the colored reaction was developed by incubating with 2,2'-azino-bis(3-ethylbenzthiazoline-6-sulfonic acid) (ABTS) - liquid substrate for horseradish peroxidase (Sigma). Absorbance at $405 \mathrm{~nm}$ was measured after 30-45 min in an ELISA reader. As controls, wells with no serum were used.

The assay was performed in triplicate for each serum and the results are presented as the mean difference between the reading in optical density units (OD) in experimental wells and controls.

Statistical analysis. Spearman rank correlations or generalized linear modeling was used to assess differences in absorbance levels (using log-transformed values of absorbance) for a more quantitative assessment of factors affecting antiMUC-1 antibody production. Combinations of factors were examined to identify the best cumulative index of experiences associated with the likelihood of having antibodies.

The MUC-1 assay methods were compared using oneway analysis of variance (ANOVA) and $t$-student test for dependent and independent samples. The statistical significance of the measured differences was determined using an alpha index of 0.05 .

\section{Results}

The mean levels of sMUC-1 in all patients with MM and healthy individuals showed no statistically significant differences $(0.81 \pm 0.25$ vs. $0.78 \pm 0.05$; $\mathrm{p}=0.38)$. According to the ISS, we found significant differences of mean sMUC-1 levels between the first stage of the disease $(0.63 \pm 0.26)$ and the third $(0.93 \pm 0.24 ; p=0.03)$, but not the second $(0.80 \pm 0.22$; $\mathrm{p}=0.08)$. Based on previous responses in over 500 cancer cases and controls, absorbance reactions at $>1.0$ were scored as high [5]. The study revealed that patients with more advanced disease were most likely to have an elevated baseline MUC-1 [first stage, zero of 5 patients $(0 \%)$; second stage, eight of 20 $(40 \%)$ and third stage, 12 of $25(48 \%) ; p=0.1]$.

In comparison with the levels of healthy subjects, high levels of sMUC-1 were found in 20/50 (40\%) MM patients. In the healthy control group, only two subjects had high levels (4\%), and this difference was statistically significant ( $\mathrm{p}=0.001)$.

From the various parameters of MM, we found statistical correlation with elevated lactate dehydrogenase $(r=0.71 ; p=0.03)$ and the level of $\mathrm{IgG}$ in

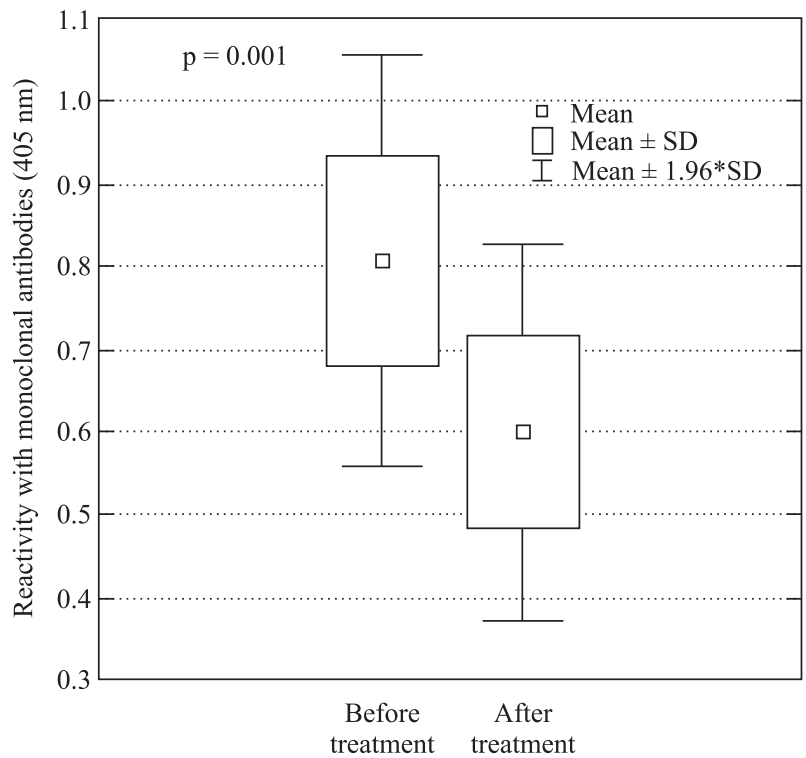

Figure 1. Reactivity with monoclonal antibodies (sMUC-1) at $405 \mathrm{~nm}$ in multiple myeloma patients before and after treatment

groups of patients with MM IgG at every stage of disease $(r=0.87 ; p=0.001)$. According to the ISS, in the third group we found significant correlation between percentage of bone marrow plasma cells from the smear of the bone marrow ( $\mathrm{r}=0.73$; $\mathrm{p}=0.01$ ), but not with the immunohistochemically detected percentage of plasma cells from biopsy samples $(\mathrm{r}=0.54 ; \mathrm{p}=0.375)$.

In univariate analysis, we found a strong tendency for higher response rate $(\mathrm{RR}=$ patient with partial response and complete remission) among patients with normal levels of MUC-1 (RR - $40 \%$ vs. $16 \%$ for those with elevated level; $p=0.072)$. After the treatment, levels of sMUC- 1 from all MM patients were statistically lower than the levels before the treatment $(0.81 \pm 0.25$ vs. $0.59 \pm 0.3 ; p=0.001)$ (Figure 1$)$. A decrease in the level of sMUC-1 was also found in the group of patients with initially higher levels of sMUC-1 and in those with normal levels. Decreased levels of sMUC-1 in groups, based on ISS, were: first stage $(0.63 \pm 0.26$ vs. $0.42 \pm 0.13 ; p=0.0006)$, second stage $(0.80 \pm 0.22$ vs. $0.56 \pm 0.2 ; p=0.00072)$, and third stage $(0.93 \pm 0.24$ vs. $0.72 \pm 0.13 ; \mathrm{p}=0.01)$. Decreased levels of sMUC-1 after treatment in a group of patients with initially elevated levels of MUC-1, were statistically lower than in a group of patients with initially lower levels of sMUC-1 (21\% to $42,6 \%$; $\mathrm{p}=0.05)$. We did not find statistically significant differences between the type of chemotherapy and the level of sMUC-1 after the treatment.

At 37 months median of follow-up, we found a statistically significant difference between patients 
with normal versus elevated sMUC-1 in terms of progression-free survival (median 12 months vs. 8.1 months; $p=0.03$ ).

\section{Discussion}

Multiple myeloma is an 'indolent' disease. Even though its disease progression seems to be somewhat slower and less aggressive than other hematologic malignancies, a complete cure can hardly be achieved by standard chemotherapy or even by bone marrow transplantation. MUC-1 is of particular interest because it is a diagnostic marker that exists as an integral membrane glycoprotein and as shed forms, and because it is a therapeutic target for both cell-mediated and antibody-based immunotherapeutic strategies.

Although most of the work to date with this target antigen has been in breast and pancreatic cancers, a few reports have shown that sMUC- 1 can be detected in the serum of MM patients [24]. The presence of MUC-1 on the cell surface of MM cells has been identified, as well, by several investigators [25-27]. It has also been shown that patients with MM have high peripheral blood and bone marrow levels of MUC-1, and that the latter directly correlate with tumor mass [27]. Treon et al. [27] found that dexamethasone (Dex) induces MUC-1 expression on MM cell lines. On the other hand, Durum et al. [28] found that stimulation of MM cells with IL-7 is associated with increases in MUC-1 expression at the mRNA and protein levels.

Similarly to the studies of Luminari et al. [26] and Mileshkin et al. [29], we were unable to demonstrate a statistically significant difference between the sMUC-1 from MM patients and healthy individuals, but we found statistical differences between the first and third stages. Furthermore, like those studies, we did not find a correlation between sMUC-1 levels and various parameters of $\mathrm{MM}$, but we confirmed the statistically significant correlation, observed by Luminari et al. [26], between elevated sMUC-1 and elevated lactate dehydrogenase but not with anemia. Increased sMUC-1 level is correlated with features associated with high tumor burden, such as high serum lactate dehydrogenase. Similarly to Treon et al. [30] and Luminari et al. [26], we demonstrated that patients with more advanced disease were most likely to have an elevated baseline sMUC-1. Although these malignant cells may be the source of sMUC-1, it also remains possible that MM cells trigger release of sMUC- 1 from other cells which contribute, at least in part, to the elevated sMUC-1 levels found in MM patients. Our findings showed that sMUC-1 levels are elevated in plasma patient samples, especially in a group of patients in ISS third stage.
Furthermore, Luminari et al. [26], based on the fact that high levels of sMUC-1 are very frequently associated with a diagnosis of plasma cell leukemia and indicate a possible relationship between them and tumor malignancy (cell proliferation through growth factor receptor, $\beta$-catenin and $\mathrm{ER} \alpha$, and suppressing apoptosis through the regulation of JNK, NF- $\kappa \mathrm{B}$, HSP90, and extrinsic apoptotic pathways), have suggested that the initial level of sMUC-1 may have a role in tumor progression of MM patients [20, 31]. In our study, at 37 months median of follow-up, we found a statistically significant difference between patients with normal versus elevated sMUC- 1 in terms of progression-free survival. These results suggest that sMUC-1 can be considered to be an independent prognostic factor in MM patients.

Therefore, antibodies against MUC-1 may be potential candidates for patient-adapted, antibody-mediated immunotherapy in MM. In addition, this observation might also contribute to cellular immunity, because sMUC-1 specific T cells against under-glycosylated forms have been demonstrated in MM patients [32].

If the aforementioned mucin interactions are required to promote tumor progression, then it could be useful to target these interactions for the treatment of cancer. Furthermore, the disruption of these interactions would elucidate the contribution of each process, and the intervention of these pathways may be helpful in controlling tumor progression.

\section{References}

1. Patton S, Gendler SJ, Spicer AP. The epithelial mucin, Muc1, of milk, mammary gland and other tissues. Biochim Biophys Acta. 1995;1241:407-424.

2. McGuckin MA, Hurst TG, Ward BG. Heterogeneity in production, secretion, and glycosylation of Muc1 epithelial mucin by primary cultures of ovarian carcinoma. Int J Cancer. 1995;63:412-418.

3. Burchell J, Gendler S, Taylor-Papadimitriou J et al. Development and characterization of breast cancer reactive monoclonal antibodies directed to the core protein of the human milk mucin. Cancer Res. 1987;47:5476-5482.

4. Porowska H, Paszkiewicz-Gadek A, Lemancewicz D, Bielawski T, Wołczyński S. Effect of brefeldin A on membrane localization of MUC1 mucin and adhesive properties of cancer cells. Neoplasma. 2008;55:305-311.

5. Vizcarra E, Lluch A, Cibrian R et al. Value of CA 15.3 in breast cancer and comparison with CEA and TPA: a study of specificity in disease-free follow-up patients and sensitivity in patients at diagnosis of the first metastasis. Breast Cancer Res Treat. 1996;37:209-216.

6. Cramer DW, Titus-Ernstoff L, McKolanis JR et al. Conditions associated with antibodies against the tumor-associated antigen MUC1 and their relationship to risk for ovarian cancer. Cancer Epidemiol Biomarkers Prev. 2005;14:1125-1131.

7. Hayes DF, Sekine H, Ohno T et al. Use of a murine monoclonal antibody for detection of circulating plasma DF3 antigen levels in breast cancer patients. J Clin Invest. 1985;75:1671-1678. 
8. Takahashi T, Makiguchi Y, Hinoda Y et al. Expression of Muc1 on myeloma cells and induction of HLA unrestricted CTL against Muc1 from a multiple myeloma patient. J Immunol. 1994;153:2102-2109.

9. Barnd DL, Lan MS, Metzgar RS, Finn OJ. Specific MHC unrestricted recognition of tumor associated mucins by human cytotoxic T cells. Proc Natl Acad Sci USA. 1989;86:7159-7163.

10. Ioannides $\mathrm{CG}$, Fisk B, Jerome KR et al. Cytotoxic T cells from ovarian malignant tumors can recognize polymorphic epithelial mucin core peptides. J Immunol. 1993;151:3693-3703.

11. Hayes DF, Silberstein DS, Rodrique SW, Kufe DW. DF3 antigen, a human epithelial cell mucin, inhibits adhesion of eosinophils to antibody coated targets. J Immunol. 1990;145:962-970.

12. van de Wiel-van Kemenade E, Ligtenberg MJ, de Boer AJ et al. Episialin (Muc1) inhibits cytotoxic lymphocyte-target cell interaction. J Immunol. 1993;151:767-776.

13. Irimura T, McIsaac AM, Carlson DA et al. Soluble factor in normal tissues that stimulates high-molecular-weight sialoglycoprotein production by human colon carcinoma cells. Cancer Res. 1990;50:3331-3338.

14. Fujita K, Denda K, Yamamoto M et al. Expression of MUC1 mucins inversely correlated with post-surgical survival of renal cell carcinoma patients. B Cancer. 1999;80:301-308.

15. Teoh $\mathrm{G}$, Chen L, Urashima $\mathrm{M}$ et al. Adenovirus vector based purging of multiple myeloma cells. Blood. 1998; 92:4591-601.

16. Croce CM. Oncogenes and cancer. N Engl J Med. 2008;358: 502-511.

17. Li Y, Liu D, Chen D, Kharbanda S, Kufe D. Human DF3/ /MUC1 carcinoma-associated protein functions as an oncogene. Oncogene. 2003;22:6107-6110.

18. Moniaux N, Chaturvedi P, Varshney GC et al. Human MUC4 mucin induces ultrastructural changes and tumorigenicity in pancreatic cancer cells. Br J Cancer. 2007;97:345-357.

19. Bafna S, Singh AP, Moniaux N, Eudy JD, Meza JL, Batra SK. MUC4, a multifunctional transmembrane glycoprotein, induces oncogenic transformation of NIH3T3 mouse fibroblast cells. Cancer Res. 2008;68:9231-9238.

20. Wei X, Xu H, Kufe D. Human MUC1 oncoprotein regulates p53-responsive gene transcription in the genotoxic stress response. Cancer Cell. 2005;7:167-178.
21. MacLean GD, Reddish MA, Longenecker BM. Prognostic significance of preimmunotherapy serum CA27.29 (MUC1) mucin level after active specific immunotherapy of metastatic adenocarcinomas patients. J Immunother. 1997;20:70-79.

22. Gertz M, Giralt S, Jagannath S, Vesole D. Criteria for evaluating disease response and progression in patients with multiple myeloma treated with high-dose therapy and haematopoietic stem cell transplantation. BrJ Haematol. 1998,102: 1115-1123.

23. Radziejewska I, Borzym-Kluczyk M, Namiot Z, Stefańska E. Glycosylation of mucins present in gastric juice: the effect of helicobacter pylori eradication treatment. Clin Exp Med. 2011;11:81-88.

24. Paydaş S, Sahin B, Gönlüşen G, Hazar B, Zorludemir S. MUC1 expression in plasmacytoma. Leuk Res. 2001;25:221-225.

25. Takahashi T, Makiguchi Y, Hinoda $Y$ et al. Expression of MUC1 on myeloma cells and induction of HLA-unrestricted CTL against MUC1 from a multiple myeloma patient. J Immunol. 1994;153:2102-2109.

26. Luminari S, Goldaniga $\mathrm{M}$, Ceccherelli $\mathrm{F}$ et al. Prevalence and prognostic significance of sMUC-1 levels in plasma cell dyscrasias. Br J Haematol. 2003;121:772-774.

27. Treon SP, Mollick JA, Urashima M et al. Muc-1 core protein is expressed on multiple myeloma cells and is induced by dexamethasone. Blood. 1999;93:1287-1298.

28. Durum SK, Aiello FB. Interleukin-7 induces MUC1. Cancer Biol Ther. 2003;2:194-195.

29. Mileshkin L, Prince HM, Seymour JF, Biagi JJ. Serum MUC-1 as a marker of disease status in multiple myeloma patients receiving thalidomide. Br J Haematol. 2003;123:747-748.

30. Treon SP, Shima Y, Grossbard ML et al. Treatment of multiple myeloma by antibody mediated immunotherapy and induction of myeloma selective antigens. Ann Oncol. 2000; 11:107-111.

31. Bafna S, Kaur S, Batra SK. Membrane-bound mucins: the mechanistic basis for alterations in the growth and survival of cancer cells. Oncogene. 2010;29:2893-2904.

32. Noto H, Takahashi T, Makiguchi $\mathrm{Y}$ et al. Cytotoxic T lymphocytes derived from bone marrow mononuclear cells of multiple myeloma patients recognize an underglycosylated form of MUC1 mucin. Int Immunol. 1997;9:791-798.

Submitted: 11 April, 2011 Accepted after reviews: 18 September, 2011 